\title{
MoonWalker, a Lower Limb Exoskeleton able to Sustain Bodyweight using a Passive Force Balancer
}

\author{
Sébastien Krut, Michel Benoit, Etienne Dombre and François Pierrot
}

\begin{abstract}
This paper presents MoonWalker, a lower limb exoskeleton able to sustain part of a user's bodyweight. This orthosis can be used for rehabilitation, to help people having weak legs, or to help those suffering from a broken leg, to walk. It can also be used as an assistive device helping people carrying heavy loads. Its main characteristic is that a passive force balancer provides the force to sustain bodyweight. An actuator is also required, but is used only to shift that force the same side as the leg in stance. Consequently, MoonWalker requires very low energy to work on flat terrains. That motor can provide also a part of the energy to climb stairs or slopes. We believe that this approach can help improving energetic autonomy of lower limb exoskeletons.
\end{abstract}

Keywords - Exoskeleton, orthosis, passive force balancer.

\section{INTRODUCTION}

$\mathrm{R}$ ESEARCH in actuated exoskeleton devices began truly in the late 1960s [1]. Exoskeletons are defined as standalone anthropomorphic active mechanical devices that are "worn" by an operator and work in concert with the operator's movements. This paper will focus on lower-limb exoskeletons only.

Exoskeletons are mainly used to increase performance of able-bodied wearer [2] (e.g. for military applications), and to help disabled people to retrieve some motion abilities [3] (such exoskeletons are called "active ortheses" in the medical field).

The Lokomat [4], used in gait-recovery therapies, constitutes another category of exoskeletons, as it is neither portable nor stand-alone.

Let's cite the most famous lower-limbs exoskeletons up-todate and discuss their features:

- The BLEEX from U.C. Berkeley [2] was designed to help soldiers to carry heavy packs. Each leg is actuated by three hydraulic cylinders and can provide upwards and forward/reverse assistance. The control approach is based on feet pressure signals.

- ReWalk [3] developed at Haifa University, Israel, is a fully actuated electrical exoskeleton, allowing paraplegic people to walk using crutches. It follows a gait pattern copied by

Manuscript received September 15, 2009.

S. Krut, M. Benoit, E. Dombre and F. Pierrot are with the Montpellier Laboratory of Informatics, Robotics, and Microelectronics (LIRMM in French), a cross-faculty research entity of the Montpellier 2 University (UM2) and the French National Center for Scientific Research (CNRS), 161 rue Ada, 34392 MONTPELLIER CEDEX 5, FRANCE.

Corresponding author: Sébastien Krut; phone/fax: +33 4674185 88; email: krut@lirmm.fr. the patient limbs.

- HAL-3 [6] developed at Tsukuba University is targeted for both performance augmenting and rehabilitative purposes. Actuation is electrical. Its control is based on EMG signals that are processed for the exoskeleton to copy legs motions and to amplify forces.

- The Walking-Assist from Honda [7] looks like an ergonomic stool equipped with robotized legs. It helps by supporting bodyweight to reduce the load on the user's legs while walking, going up and down stairs, and in semicrouching positions. It uses electrical actuators and control relies on feet pressure signals. Assistance is only provided in the upward direction, and users have to move their leg forward to make the device walk. This choice resulted form a compromise: keeping the device simple while offering enough assistance to reduce user's muscular fatigue for daily tasks.

In this paper a new exoskeleton, called MoonWalker, is presented (see Fig. 1). Contrary to all above mentioned exoskeletons, MoonWalker is quasi-passive, requiring very low energy to work. In this sense, it is very comparable with the elastic exoskeleton presented in [8] that only uses friction-lock clutches for control. However this elastic exoskeleton uses one controlled spring per leg whereas MoonWalker uses a single force balancer coupled for the two legs.

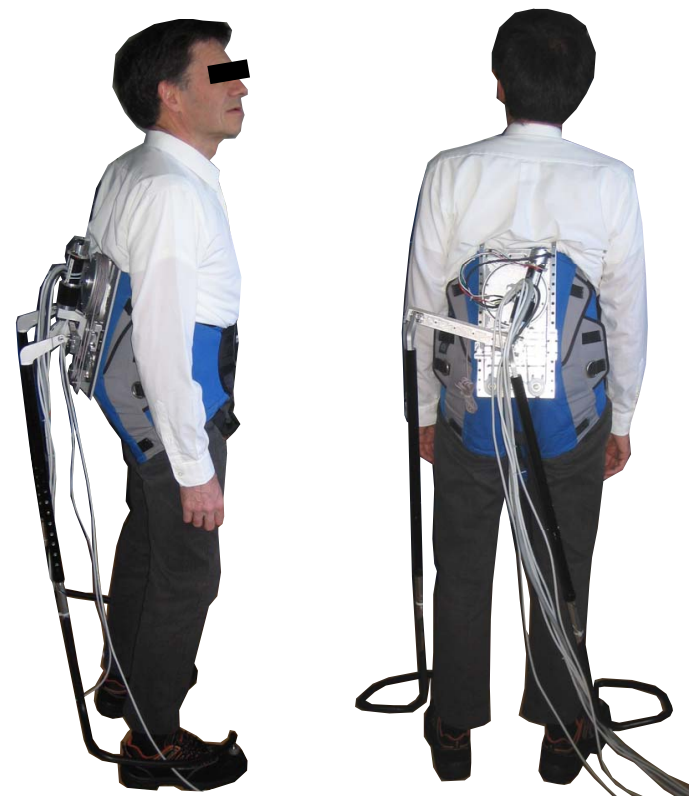

Fig. 1. Pictures of MoonWalker, a lower-limb exoskeleton able to sustain part of user's weight based on passive gravity compensation 
It offers the same kind of assistance the Walking-Assist from Honda does: it exerts an upward force on the user's pelvis to reduce the forces supported by his legs. The main difference consists in the way it is actuated: weight is compensated using a passive force balancer, and a lowpower actuator is used for control, loading the exoskeleton on the side where the user's leg is in stance, and lowering it on the side where the user's leg is in swing. This strategy allows increasing energy autonomy, which is the weak point of most actual active exoskeletons.

Section II describes the concept of MoonWalker. Section III is about control. Section IV describes the prototype, and shows some experimental results. Some possible improvements for that device are proposed in the conclusion.

\section{DESIGN CONCEPT}

\section{A. Main concept}

The main idea, when designing MoonWalker, was to propose an exoskeleton able to exert an upward vertical force on the user's pelvis in order to lighten the forces in his/her legs due to bodyweight. Doing that would reduce the load on his/her leg muscles and joints in the hip, knee, and ankle (another approach to achieve this goal is that in [9] which uses a gravity balancing leg orthosis connected to a trolley). Hence, it could benefit disabled persons with weak legs, or suffering from a broken leg. It could also benefit able-bodied people that must remain in uncomfortable semicrouching positions for long times, or wiling to carry heavy loads, by supporting that extra load. Its name, MoonWalker, was chosen as the user's legs feel only a part of body weight, giving the impression that gravity is reduced, such as on the Moon.

Another important idea when designing MoonWalker was that, to offer that assistance on flat terrains, a passive device could be used as the gravitational potential energy of the bodyweight remains almost the same. This idea is illustrated on Fig. 2, where the user is connected to a trolley via a force balancer which exerts a constant upwards vertical force on the pelvis, lightening the forces due to his bodyweight on his legs. A force balancer is inserted between the trolley and user's pelvis, instead of a rigid link, to allow some vertical motion, the one naturally generated when walking. That device is very similar to baby jumpers. The only difference is that, baby jumpers use classical springs or rubber bands, whereas on that device, a force balancer is used (acting like a spring exerting the same reaction force whatever its extension is). This results in a smooth assistance that avoids bouncing inconsiderately.



Fig. 2. Bodyweight compensation principle illustrated with a trolley equipped with a force balancer

Next step is to find a solution to get rid of the trolley. We thought of a way to replace it by two sticks running along each leg (see Fig. 3) in order to provide an exoskeleton, that is to say, a mechanical device that is "worn" by the patient.

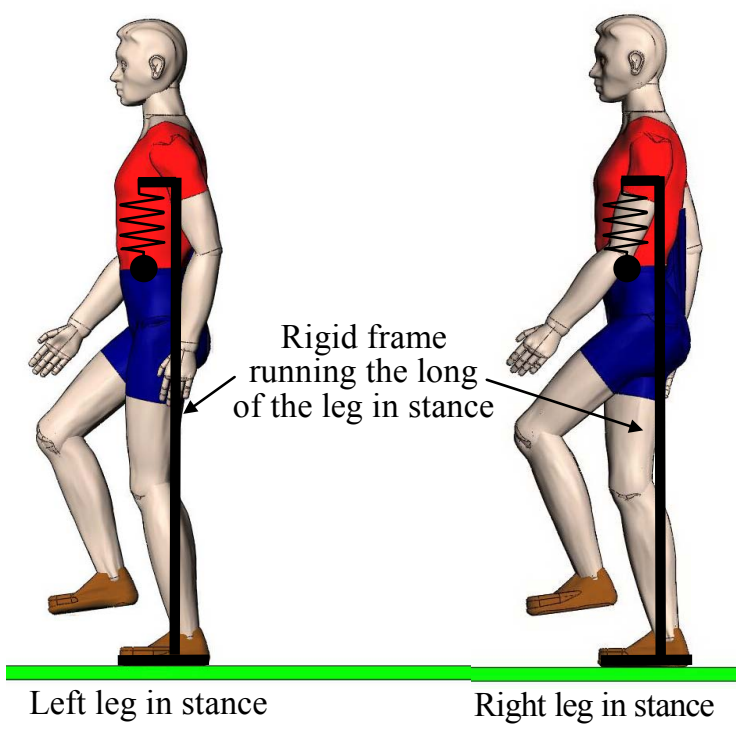

Fig. 3. Bodyweight compensation principle illustrated with two poles running along each leg

The difficulty is that the force balancer has to be connected to the stick running on the side of the leg in stance only, in order to let the other leg swing freely with the other stick being disconnected from the force balancer. To achieve this goal, a driven device able to share the force produced by the balancer on the two sticks in a continuous manner (from $0 \%$ to $100 \%$ right/left leg force repartition) was imagined. 


\section{B. Force sharing device description}

The force sharing device, able to share the force exerted by the balancer between the two sticks in a continuous manner, is depicted in Fig. 4.


Fig. 4. Kinematic and implementation schemes of MoonWalker. S, U, P, R stand for Spherical, Universal, Prismatic and Revolute joints, respectively.

It consists in a beam connected at its ends to the two sticks using passive Universal joints (U). The beam is actuated to slide from right to left using an actuated Prismatic joint (P). The linear actuator is connected using a passive Revolute joint $(\mathrm{R})$ to a vertical cart. That cart is guided relatively to user's pelvis using a passive $\mathrm{P}$ joint, and is connected to the force balancer attached to user's pelvis.

The sticks are connected to rigid shoes in order to transmit the forces passing through the sticks to the ground using Spherical joints (S). U and $\mathrm{S}$ joints where selected for the sticks ends in order to transmit the balancer force to the ground and to allow the shoes to move freely. Note that, with such a device, the forces transmitted to the ground are not purely vertical, but are lined up with the line going from the center of the spherical joints to the center of the universal joints. In practice, this is not a big issue while the main components of these forces remain vertical. This phenomenon also occurs on the Walking-Assist from Honda [7]. However, thanks to the use of arc-shaped guide tracks located below user's seat, the assisting forces are always directed toward the user's pelvis, which corresponds roughly to the user's centre of gravity.

Fig. 5 shows the force sharing device in two different configurations. When the two legs are in stance, the beam is centered sharing the balancer force equally on both sticks. When the left leg is in stance and the right leg is in swing, the beam needs to be shifted to the right. Hence, for that configuration, the force balancer does not exert any force on the right stick, which allows the right leg to move freely. Additionally, the left stick transmits all the force delivered by the balancer to the ground. The opposite configuration (beam shifted to the left) allows the opposite walking pattern: right leg in stance and left leg in swing.

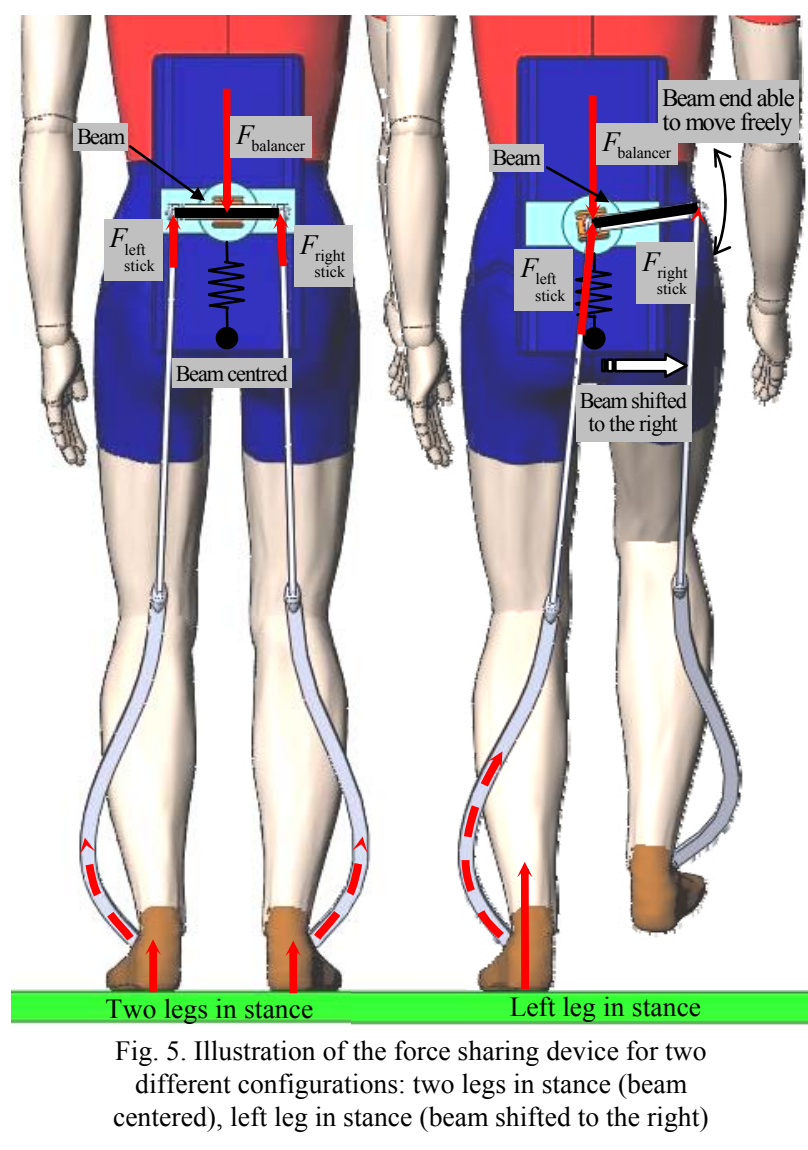

\section{Force sharing device modeling}

Modeling of the force sharing device is done to derive the relation between the beam position and the repartition of the balancer force on the two sticks. The force sharing device is modeled according to Fig. 6 .



Fig. 6. Scheme of the force sharing device: the force produced by the balancer is shared between the right and the left sticks depending on $x$. Pr. and Rev. stand for Prismatic and Revolute joints, respectively.

For simplification purpose, computation is done in the frontal plane, in $2 \mathrm{D}$ only. $R=<\mathrm{O}, \vec{i}, \vec{j}>$ is defined to be the base attached to the vertical cart such that $\vec{j}$ is lined up with the direction of the force delivered by the balancer ( $\vec{F}_{\text {balancer } \rightarrow \text { cart }}=-F_{\mathrm{b}} \vec{j}$ ) on the system. $\mathrm{O}$ is its application point. Note that, as the force delivered by the balancer is not transmitted directly to the beam, but through the serial chain made with the passive R-P-R joints, only the component of that force being lined-up with $\vec{v}$, the vector perpendicular to 
the direction $\vec{u}$ of the $\mathrm{P}$ joint that guides the beam, is transmitted to the beam:

$$
\vec{F}_{\text {cart } \rightarrow \text { beam }}=\left(\vec{F}_{\text {balancer } \rightarrow \text { cart }} \cdot \vec{v}\right) \vec{v}
$$

where $\cdot$ denotes the scalar product.

As sticks are very long, it is assumed that they won't tilt much in the coronal plane, and that they remain lined up with the force balancer. Consequently, the forces they can transmit always remain in the same direction: $\vec{F}_{\text {left stick }}=F_{1} \vec{j}$ and $\vec{F}_{\text {right stick }}=F_{\mathrm{r}} \vec{j}$. L and $\mathrm{R}$ are their application points (see Fig. 6). $x$, the displacement of the center $\mathrm{C}$ of the beam satisfies $\overrightarrow{\mathrm{OC}}=x \vec{u}$, where $x \in[-l, l]$ and $2 l$ is the beam length. Consequently, $\overrightarrow{\mathrm{OL}}=(l-x) \vec{u}$ and $\overrightarrow{\mathrm{OR}}=(l+x) \vec{u}$. The force $\vec{F}_{\text {actuator }}$ provided by the actuator for the system to remain in static equilibrium, is collinear with $\vec{u}$, the direction vector of the prismatic joint that guides the beam: $\vec{F}_{\text {actuator }}=F_{\text {a }} \vec{u}$.

Applying the static equilibrium principle on the beam at point $\mathrm{O}$ leads to:

$$
\left\{\begin{array}{c}
\vec{F}_{\substack{\text { balancer } \\
\text { transmitted }}}+\vec{F}_{\text {actuator }}+\vec{F}_{\text {left }}+\vec{F}_{\text {stick }}=\overrightarrow{0} \\
\overrightarrow{\text { stick }} \\
\overrightarrow{\mathrm{OL}} \times \vec{F}_{\text {left }}+\overrightarrow{\mathrm{OR}} \times \vec{F}_{\text {stight }}=\overrightarrow{0}
\end{array}\right.
$$

where $\times$ denotes the cross-product.

This leads to:

$$
\left\{\begin{array}{c}
F_{\mathrm{a}}=-F_{\mathrm{b}} \sin (\theta) \\
F_{1}=\frac{1-\varepsilon}{2} F_{\mathrm{b}}, F_{\mathrm{r}}=\frac{1+\varepsilon}{2} F_{\mathrm{b}}
\end{array}\right.
$$

with $\varepsilon=x / l$ being the normalized displacement of the beam $(\varepsilon \in[-1,1])$ and $\theta$ is the tilting angle of the beam ( $\theta=\widehat{\vec{i}, \vec{u}})$. Note that relations (5) do not depend on the value of $\theta$, meaning that the way the beam is tilted does not modify force repartition.

\section{Power consumption estimation}

To estimate power consumption, the mechanical energy required by the actuator to move the beam is computed:

$$
\delta W=-\vec{F}_{\text {actuator }} \cdot \overrightarrow{d x}=-F_{\mathrm{a}} d x=F_{\mathrm{b}} \sin (\theta) d x
$$

where $\delta W$ denotes the infinitesimal work that the actuator needs to provide a small displacement of $d x$ length. Assuming that when the beam is moved, $\theta$ remains constant, integrating relation (6) for $x$ going from left to right leads to:

$$
W=\int_{x=-l}^{x=l} \delta W=F_{\mathrm{b}} \Delta h
$$

with $\Delta h=2 l \sin (\theta)$ being the vertical elevation of the cart. Note that this computation does witness mechanical energy only. Energy consumption estimation might be quite different from this value, as system efficiency is not taken into account, and as the power system might not be able to benefit from regenerative energy coming from the actuator. However the interpretation of result (7) shows that:

(i) When the users walk of flat terrains, a transition from one foot to the other does not modify vertical the elevation of the cart the height $(\Delta h \simeq 0)$. Hence power consumption is very low, as the power supply as to deliver only the energy to overcome friction and dynamics effects.

(ii) When the user climbs a slope or a stair of height $\Delta h$, the power supply has to provide the energy corresponding to $E=F_{\mathrm{b}} \Delta h$ only. During descents, regenerative energy provided by the actuator can be sent back to the power supply.

The rest of the energy required to sustain part of the user bodyweight, is delivered by the passive force balancer, and does not require any energy coming from the power supply.

\section{CONTROL}

Control of MoonWalker is very simple: it consists in measuring the way the user shares his reaction force on the ground between his two legs and to drive the force sharing device accordingly to distribute the balancer force between the two sticks in the same proportion.

Measuring the way the user shares his reaction force on the ground between his two legs is done using pressure sensors located below his two feet. Driving the force sharing device is done controlling an actuator that can move the main beam right and left. The global control scheme is given in Fig. 7.

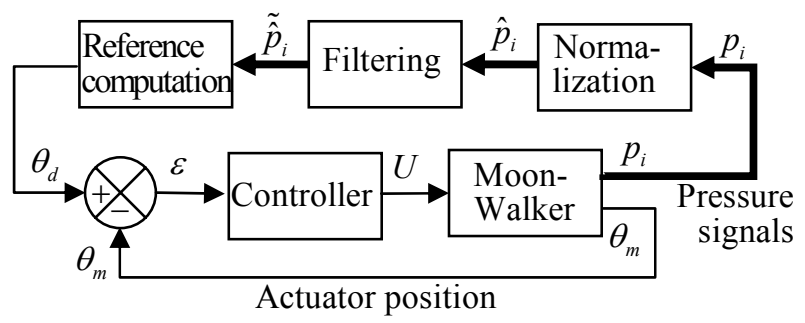

Fig. 7. Control scheme of MoonWalker

Three pressure sensors where placed in each shoe below user's feet: one under the heel and two on front. As we encountered some practical difficulties with the FSR (Force Sensing Resistors) raw signals had to be preprocessed (normalized and filtered) in order to obtain acceptable data.

\section{A. Preprocessing raw pressure sensors data}

Normalizing of pressure signals was done to get the very same response from every sensor:

$$
\hat{p}_{i}=\frac{p_{i}-p_{i}^{\min }}{p_{i}^{\max }-p_{i}^{\min }}
$$

where $p_{i}, i \in\{1, \ldots, 6\}$ represents the signals recorded from FSR number $i, p_{i}^{\min }$ and $p_{i}^{\max }$ represent their extreme values obtained during a calibration phase, $\hat{p}_{i}$ represent the normalized values $\left(\hat{p}_{i} \in[0,1]\right)$.

Filtering was done using a simple digital filter, computed applying the classical Z-transformation to the first order 
low-pass filter, which Laplace's expression is given by $G(s)=\omega_{0} /\left(s+\omega_{0}\right):$

$$
\tilde{\hat{p}}_{i}[k]=a\left(\omega_{0} T_{e} \hat{p}_{i}[k]+\tilde{\hat{p}}_{i}[k-1]\right)
$$

where $\hat{p}_{i}[k]$ represents the normalized pressure computed at time step $k, \tilde{\hat{p}}_{i}[k]$ and $\tilde{\hat{p}}_{i}[k-1]$ represent the filtered normalized pressure computed at time steps $k$ and $k-1, \omega_{0}$ is the cutoff pulsation corresponding to the cutoff frequency $f_{0}\left(\omega_{0}=2 \pi f_{0}\right), T_{e}$ is the sampling period, and $a$ is the digital filter constant $\left(a=1 /\left(1+\omega_{0} T_{e}\right)\right)$.

Doing this preprocessing step led to $\tilde{\hat{p}}_{i}$ being reliable measures of the pressures exerted by the users legs on he ground.

\section{B. Generating the input value for the beam position}

Next step was to compute the "global" pressures exerted by the left and right foot on the ground, $p_{\text {left }}$ and $p_{\text {right }}$. That step was not investigated much and the mean value of the sensors for each foot was taken:

$$
\left\{\begin{array}{l}
p_{\text {left }}=\left(\tilde{\hat{p}}_{1}+\tilde{\hat{p}}_{2}+\tilde{\hat{p}}_{3}\right) / 3 \\
p_{\text {right }}=\left(\tilde{\hat{p}}_{4}+\tilde{\hat{p}}_{5}+\tilde{\hat{p}}_{6}\right) / 3
\end{array}\right.
$$

where $\tilde{\hat{p}}_{1}, \tilde{\hat{p}}_{2}, \tilde{\hat{p}}_{3}$ correspond to the left foot and $\tilde{\hat{p}}_{4}, \tilde{\hat{p}}_{5}$, $\tilde{\hat{p}}_{6}$, to the right foot.

The linear function $\eta \in[-1,1]$, representing the way force is shared between the two legs, was built this way:

$$
\eta=\frac{p_{\text {right }}-p_{\text {left }}}{p_{\text {right }}+p_{\text {left }}} \in[-1,1]
$$

$\eta=-1$ meaning that all pressure is exerted on the left foot, and $\eta=1$ that all pressure is exerted on the right foot. Driving the force sharing device is done based on the value of $\eta$.

The force sharing device is controlled in position using a simple PID acting on the actuator joint coordinate $\theta_{d} . \theta_{d}$ is linked to $\varepsilon=x / l \quad(\varepsilon \in[-1,1]) \quad$ the normalized displacement of the beam, using the simple affine relation:

$$
\theta_{d}=\theta^{\min }+\frac{1+\varepsilon}{2}\left(\theta^{\max }-\theta^{\min }\right)
$$

where $\theta^{\min }$ and $\theta^{\max }$ are the extreme values for $\theta_{d}$ corresponding to the left and right beam ends $\left(\theta_{d} \in\left[\theta^{\min }, \theta^{\max }\right]\right)$.

Achieving our goal (that is to say, moving the device the proper way according to user's force repartition) can be done easily by choosing $\varepsilon=\eta$, and computing the reference $\theta_{d}$ of the PID controller using (13). However, to be sure that the leg in swing would be totally unloaded even if some small signals are measured by the pressure sensors of the leg in swing, a symmetrical monotonic function $f, \varepsilon=f(\eta)$, with two steps of length $\tau$ at its ends, was built. The part of the function between $-1+\tau$ and $1-\tau$ was chosen to be a degree-3 polynomial that would join the two steps in a tangent manner (see Fig. 8), leading to a continuously differentiable $C^{1}$ function for $f$ :

$$
\left\{\begin{array}{c}
\text { if } \eta \leq-1+\tau \text {, then } \varepsilon=-1 \\
-1+\tau<\eta<1-\tau \text {, then } \varepsilon=-\frac{1}{2(1-\tau)^{3}} \eta^{3}+\frac{3}{2(1-\tau)} \eta \\
\text { if } \eta \geq 1-\tau, \text { then } \varepsilon=1
\end{array}\right.
$$

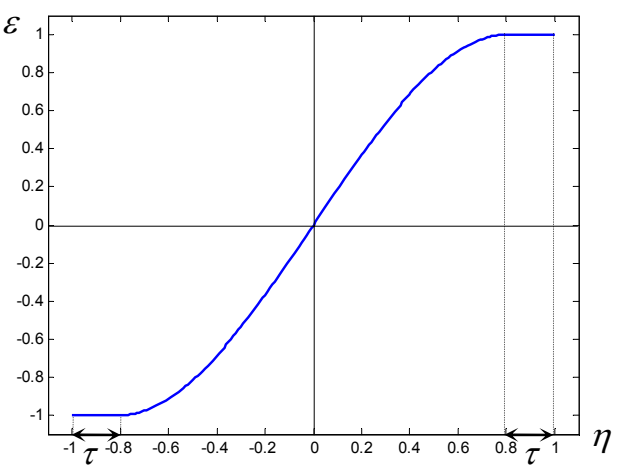

Fig. 8. Illustration of the function linking feet pressure repartition and the position of the beam of the force sharing device.

\section{PROTOTYPE DESCRIPTION AND EXPERIMENTS}

A prototype of MoonWalker was built to test the effectiveness of the concept (see Fig. 1). A harness was chosen to interface the force sharing device with the user's pelvis. A simple spring balancer was selected for the force balancer (6080B from Prevost). However, due to its force limitation ( $8 \mathrm{kgF}$ maximum only), a hoist was built using a set of pulleys, to amplify to force by four (see the cable running from the vertical cart to the harness four times, in Fig. 9).



Fig. 9. CAD view of the practical implementation of the force sharing device

The maximum force that can be compensated on user's leg is than $32 \mathrm{kgF}(\sim 320 \mathrm{~N})$, which correspond to a ratio between a third an a half of a normal's user bodyweight. A rotary motor (EC-powermax 30 from Maxon Motor) equipped with a gear reducer (GP 42C from Maxon Motor) and an absolute encoder (AD36 from Hengstler) was 
selected to drive the beam thanks to a pinion and a rack. The motor, the reduction ratio of the gear box, and the diameter of the pinion were chosen in order for the beam to be able to deliver a force up to $400 \mathrm{~N}$, a force higher than the one provided by the force balancer.

The force sharing device was implemented as close as possible from user's body in order to limit as much as possible disturbing torques due to the lever arms between user's center of mass and the application points of the forces transmitted by the sticks. The sticks were curved in order not to interfere with the user's legs. The length of the beam was chosen to be $300 \mathrm{~mm}$, in order to be long enough to allow the user to climb stairs, and short enough not to collide with user's arms. Control hardware was not embedded at this stage of the project. It consists in a PC running a real time OS (RTX from Ardence), a homemade PCI IO board and a mains-powered amplifier (Accelus ASP-055-18 from Copley Controls) connected to MoonWalker using an extension lead.

Early tests demonstrate qualitatively the effectiveness of the proposed concept (see the corresponding video). The ablebodied user was able to walk easily without feeling uncomfortable due to the device. Assistance was really noticeable, when standing in semi-crouching positions, and when walking up-stairs.

\section{CONCLUSION AND FURTHER WORK}

The whole report here is the first phase of a research project aiming at offering an exoskeleton requiring very low powerconsumption. So far, conceptual design has been proven up to the functional level only. Assessment of its usefulness for patient related purposes will be done in the next future. It will be verified that the device reduces load on leg muscles and joints (in the hips, knees, and ankles) by supporting a portion of the user's bodyweight.

The main characteristic of Moonwalker is that a passive force balancer provides the force to sustain bodyweight. It is controlled using an actuator that requires very low energy to work on flat terrains, as it is used only to shift that force the same side as the leg in stance. That motor is able also to provide a part of the energy to climb stairs or slopes. We believe that this approach can help improving energetic autonomy of lower limb exoskeletons.

\section{REFERENCES}

[1] A. M. Dollar, and Hugh Herr, "Lower Extremity Exoskeletons and Active Orthoses: Challenges and State-of-the-Art," in IEEE TRO (Transactions On Robotics), Vol. 24, No. 1, February 2008.

[2] A.B. Zoss, H. Kazerooni, and A. Chu, "Biomechanical design of the Berkeley lower extremity exoskeleton (BLEEX)," in IEEE/ASME Transactions on Mechatronics, Vol. 11(2), pp. 128-138, ISSN: 10834435, April 2006.

[3] A. Goffer, "Gait-locomotor Apparatus," European patent EP1260201 (A1), Argo Medical Technologies LTD, November 27, 2002.

[4] G. Colombo, M. Joerg, R. Schreier, and V. Dietz, "Treadmill Training of Paraplegic Patients using a Robotic Orthosis," in Journal of Rehabilitation Research and Development, Vol. 37, pp. 693-700, 2000.

[5] J. Dick and E. Edwards, "Human bipedal locomotion device," U.S. Patent 5016 869, 1991.

[6] H. Kawamoto and Y. Sankai, "Power assist system HAL-3 for gait disorder person," in Proc. Int. Conf. Comput. Helping People Special Needs (ICCHP) (Lecture Notes on Computer Science), vol. 2398, Berlin, Germany: Springer-Verlag, 2002.

[7] J. Ashihara, and Y. Hiki, Walking Assistant Device, U.S. Patent 2008/0289670 A1, Honda Motor Co. LTD, November 27, 2008.

[8] M. S. Cherry, S. Kota, and D. P. Ferris, "An Elastic Exoskeleton for Assisting Human Running," in Proc. of IDETC/CIE 2009, ASME 2009 Int. Design Engineering Technical Conferences \& Computers and Information in Engineering Conf., San Diego, California, USA, August 30 - September 2, 2009.

[9] S.K. Banala, S.K. Agrawal, A. Fattah, K. Rudolph, J.P. Scholz, "A Gravity Balancing Leg Orthosis for Robotic Rehabilitation," in Proc. of ICRA'04: IEEE 2004 International Conference on Robotics and Automation, Vol.3, pp. 2474- 2479, 26 April - May 1 ${ }^{\text {st }}, 2004$. 